\title{
Assessment of Platelet Numbers and Prevalence of Thrombocytopenia among Children with Severe Malaria at a Tertiary Teaching Hospital
}

\author{
David Manukure Ansah1, Maxwell Hubert Antwi2 ${ }^{2}$, Seth Anane ${ }^{3}$, Jephthah Boateng Ashley4, \\ Adomako Francisca Serwaa ${ }^{5}$
}

${ }^{1}$ Department of Medical Laboratory Science, School of Allied Health Sciences, University of Ghana, Legon, Ghana

${ }^{2}$ Department of Clinical Laboratory, Nkenkaasu Government Hospital, Ghana Health Service, Offinso-North, Nkenkaasu, Ghana

${ }^{3}$ Department of Clinical Microbiology-Parasitology Unit, KomfoAnokye Teaching Hospital, Kumasi, Ghana

${ }^{4}$ Department of Clinical Laboratory, Bortianor Polyclinic, Ghana Health Service, Ga-South, Bortianor, Ghana

${ }^{5}$ Department of Medical Laboratory Technology, Faculty of Health and Allied Sciences, Garden City University College,

Kumasi, Ghana

Email:bg400006@yahoo.com

How to cite this paper: Ansah, D.M., Antwi, M.H., Anane, S., Ashley, J.B. and Serwaa, A.F. (2021) Assessment of Platelet Numbers and Prevalence of Thrombocytopenia among Children with Severe Malaria at a Tertiary Teaching Hospital. Journal of Biosciences and Medicines, 9, 52-63. https://doi.org/10.4236/jbm.2021.91005

Received: December 19, 2020

Accepted: January 19, 2021

Published: January 22, 2021

Copyright $\odot 2021$ by author(s) and Scientific Research Publishing Inc. This work is licensed under the Creative Commons Attribution International License (CC BY 4.0).

http://creativecommons.org/licenses/by/4.0/

(c) (i) Open Access

\begin{abstract}
Background: Thrombocytopenia plays an important role in the diagnosis of malaria in most countries because of the rapid decrease of platelets during malaria onset and also in severe forms of the disease. The study sought to assess platelet numbers and determine the prevalence of malarial thrombocytopenia among children with febrile malaria at the Korle-Bu Teaching Hospital (KBTH). Methodology: It was a cross-sectional study carried out in the Department of Child Health and the Polyclinic of KBTH from April to July 2018. A total of 100 children aged from 1 to 12 years who tested malaria positive for both thick and thin blood film and Rapid Diagnostic Tests (RDTs) were recruited into the study. Venous blood sample was obtained from the children and analyzed for platelet count using a haematology auto analyzer. Parasite speciation and quantification was used to classify the disease into mild, moderate and severe, and platelet numbers were also categorized into low, normal and high. Data obtained was then analyzed for prevalence of malarial thrombocytopenia in severe malaria. Results: The total studied participants were 100, 61 (61\%) were males whiles 39 (39\%) were females. Both severe malaria and thrombocytopenia were mostly seen in children less than 5 years of age and the males recorded more thrombocytopenia (66\%) and normal platelet numbers $34(64.2 \%)$ than the females. There was a significant higher percentage of malaria children with thrombocytosis being females
\end{abstract}


(64\%) as compared to those being males (36\%). 53 (53\%) children had normal platelet numbers, 33 (33\%) had thrombocytopenia, and 14 (14\%) had thrombocytosis. The prevalence of uncomplicated malaria and complicated malaria were $80 \%$ and $20 \%$ respectively. The overall prevalence of thrombocytopenia was $33 \%$, and the prevalence of thrombocytopenia in uncomplicated and severe malaria was $18.8 \%$ and $90 \%$ respectively. The prevalence of malaria types among children with thrombocytopenia was assessed. Out of the $33(33 \%)$ patients who had thrombocytopenia, $15(45.5 \%)$ of them had uncomplicated malaria and 18 (54.5\%) of them had complicated or severe malaria. No child was found to have thrombocytosis during complicated malaria. Conclusion: The study indicates a significant higher prevalence of thrombocytopenia in severe malaria among children at the Korle-Bu Teaching Hospital, and this could be used as a useful indicator to investigate malaria or as a diagnostic clue for malaria in children.

\section{Keywords}

Thrombocytopenia, Malaria, Plasmodium falciparum, Platelet, Korle Bu Teaching Hospital-KBTH

\section{Introduction}

Malaria is estimated to be directly responsible for about one million deaths annually worldwide, with the morbidity and mortality burden caused by malaria being nearly 3\% [1] [2] [3] Africa also accounts for $90 \%$ of the mortality burden despite progress in intensive care and antimalarial treatment and by contrast, Plasmodium falciparum is the deadliest species and the subject of most malaria related research [4] [5]. Malaria complications involve the nervous, respiratory, renal, and hematopoietic systems. The hematological changes that may occur include thrombocytopenia which is a common clinical finding in Plasmodium falciparum malaria infection and occasionally, profound thrombocytopenia may occur in severe falciparum malaria [3] [6]. However, in general, an insignificant reduction in platelet numbers is unrelated to the severity of the infection [3] [7]. Malaria causes haemostatic abnormalities that range from asymptomatic thrombocytopenia to fulminant disseminated intravascular coagulation (DIC). Early investigations suggested that the major coagulation abnormality of malaria was DIC, [8] but in recent years clinicians are recognizing that thrombocytopenia is a common and early sign of malaria infections whereas DIC is rare. Thrombocytopenia might be a useful indicator of malaria in children [6] [9] and the mechanism of thrombocytopenia in malaria is probably the consequence of several factors. Some experimental data and clinical studies have successively emphasized the role of immune factors and the destruction or sequestration of platelets, and in severe forms, platelet and erythrocyte sequestrations are frequent and thrombocytopenia is present [10]. Thrombocytopenia is a common finding 
in malaria but its correlation with the type of malaria and prognosis in context with severity of the low platelet count has not been evaluated in many studies [11] [12]. Platelets have been implicated in animal, clinical and experimental studies of malaria pathogenesis. Histopathological studies of children who have died of severe malaria showed that platelet clumps with and without infected erythrocytes are frequently found in the vasculature [13]. Infected erythrocytes may adhere to platelets and the clumps of these erythrocytes and platelets have been associated with severe malaria [14]. Therefore, there remains a paradox that, while thrombocytopenia is associated with infection and platelets have been implicated in the pathogenesis of severe malaria, most studies suggest that low counts of platelets are not associated with an adverse outcome. Without understanding this paradox, it is not possible to evaluate the true contribution of platelet to the pathogenesis of severe malaria [15]. The causes of thrombocytopenia in acute and severe malaria are also poorly understood even in Ghana. Increased platelet destruction is significant during malaria infection and the platelet lifespan is reduced during malaria, which seems to be associated with a diffuse pattern of platelet sequestration [16] [17]. The contribution of abnormalities in platelet production to thrombocytopenia during malaria infection is less clear. The study therefore sought to assess platelet numbers and prevalence of thrombocytopenia among children with severe malaria at a tertiary teaching hospital in Ghana. It again sought to provide baseline data for future reviews and molecular research to see if thrombopoietic growth factors could be used as therapy for malarial thrombocytopenia.

\section{Materials and Method}

\subsection{Study Area}

The study was carried out in the laboratories of the Department of Child Health and the Polyclinic of the Korle-Bu Teaching Hospital in the Greater Accra region Ghana from April to July 2018.

\subsection{Study Design}

This was a cross-sectional study which involved children with malaria parasite infection who were between the ages of 1 to 12 years. They were randomly selected based on clinical examination, signs and symptoms of malaria and again with prior knowledge of family history. According to the Ghana 2016 Malaria Indicator Survey [18], the malaria prevalence in children ranges from a low of $5 \%$ in Greater Accra to a high of $31 \%$ in Eastern region of Ghana. The regional prevalence of $5 \%$ in Greater Accra was used. Using 0.05 margin of error at a 95\% confidence interval, the sample size was calculated as shown below: $n=\frac{Z^{2} p(1-p)}{m^{2}}$ Where $n=$ sample size, $Z$ is reliability coefficient $=1.96, p$ is prevalence $=5 \%, m$ is margin of error $=0.05, n=73$. The sample size was therefore run to 100 to make amends for any possible sampling error of refusal to 
participate.

\subsection{Study Population}

The study involved 100 children both males and females with both complicated and uncomplicated malaria using World Health Organization (2000) criteria at the two health care facilities between the months of April and July 2018. Children between the ages of 1 to 12 years who tested positive for malaria and whose parents and guardians gave consent to participate were included in the study. Children who were receiving intensive chemotherapy or radiotherapy, diagnosed with HIV/AIDS or had any of the haematological malignancies, those with sickle cell disease and those whose guardians refused consent were totally exempted from the study.

\subsection{Sample Collection and Processing}

Three (3) milliliters of venous blood were taken from each child and were dispensed into dipotassium ethylene diamine tetra acetic acid $\left(\mathrm{K}_{2} \mathrm{EDTA}\right)$ tube. Thick and thin blood films smears were made from each sample on clean slides and labelled accordingly. The smeared slides were air-dried and were stained with Giemsa using 1 in 10 dilution followed by subsequent observation under the light microscopy using $\times 100$ oil immersion for parasite determination and count. The parasite determination and count were categorized into mild $(<1000$ parasites/ul of blood), moderate (1000 - 4999 parasites/ul of blood), high (5000 99,999 parasites/ul of blood) and hyperparasitaemia ( $\geq 100,000)$ [19]. The high and the hyperparasitaemia categories were together considered as severe malaria. Platelets count was also determined by doing full blood count on all the samples using Sysmex Xt-2000i haematology analyzer. Platelet numbers that were given out were classified into one of three categories as normal (150 - $400 \times$ $\left.10^{9} / \mathrm{L}\right)$, low-thrombocytopenia $\left(<150 \times 10^{9} / \mathrm{L}\right)$ or high-thrombocytosis $(>400 \times$ $10^{9} / \mathrm{L}$ ) depending on whether they fell within, below or above the platelet reference range.

\subsection{Statistical Analysis}

The data collected were computed and analyzed using statistical package for social sciences (SPSS20.0). Descriptive statistics such as frequency tables and pictorial statistics such as bar chart were used to summarize the data obtained. Categorical variables were presented and reported as simple frequencies with their corresponding percentages. Means with their standard deviation and median (ranges) were used to summarize continuous variables.

\section{Results}

\subsection{Gender Distribution of Platelet Numbers among Children with Malaria}

Figure 1 shows gender distribution of platelet numbers among children with 


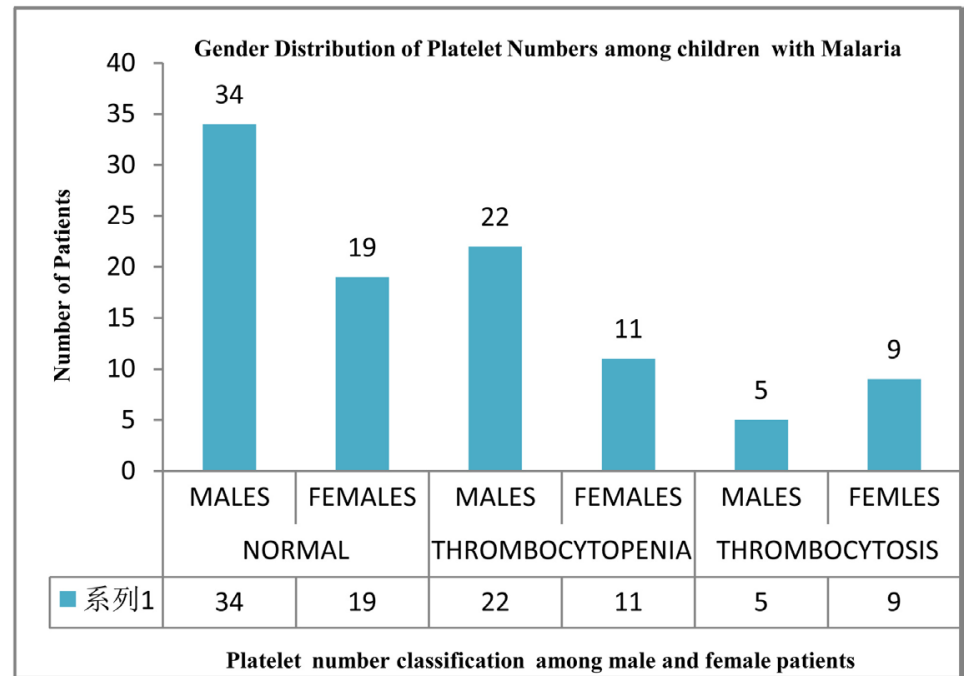

Figure 1. Gender distribution of platelet numbers among children with malaria.

malaria. The total studied participants were 100 and out of that $61(61 \%)$ were males whiles 39 (39\%) were females. 53 children with normal platelet numbers were recorded, 34 (64.2\%) were males whiles 19 (35.8\%) were females. 14 children with thrombocytosis (high platelet numbers) were also recorded and the males were 5 (36\%) whiles the females were 9 (64\%). Malaria children with thrombocytopenia (low platelet numbers) were 33, males accounted for 22 (66\%) and the females were $11(33 \%)$. There was a significant higher percentage of malaria children with thrombocytopenia being males as compared to those being females. The female children who had malaria and with thrombocytosis also recorded significant higher percentage compared to the males.

\subsection{Distribution of Platelet Numbers and Malaria Types According to Age Groups}

Table 1 shows the distribution of platelet numbers and malaria types according to the age groups of the children. Platelet numbers were classified as normal, thrombocytopenia and thrombocytosis among these age categories 1 to 4,5 to 8 and 9 to 12 years. Children with Normal platelets numbers between 1 to 4 years was 37, with thrombocytopenia and thrombocytosis between same age group was 17 and 5 respectively. Children with Normal platelets numbers between 5 to 8 years was 15, with thrombocytopenia and thrombocytosis between same age group was 12 and 7 respectively. Children with Normal platelets numbers between 9 to 12 years was 1 , with thrombocytopenia and thrombocytosis between same age group was 4 and 2 respectively. The age category that recorded frequent thrombocytopenia was seen among the 1 to 4 years followed by 5 to 8 years. Malaria types were also classified as Uncomplicated and Complicated among same age categories 1 to 4,5 to 8 and 9 to 12 years. Children with Uncomplicated malaria between 1 to 4 years was 52,5 to 8 years was 24 and 9 to 12 years was 4. Children with Complicated malaria between 1 to 4 years was 7,5 to 
Table 1. Distribution of platelet numbers and Malaria types according to age groups.

\begin{tabular}{ccccc}
\hline Category & $\mathbf{1}$ to $\mathbf{4}$ years & $\mathbf{5}$ to $\mathbf{8}$ years & $\mathbf{9}$ to $\mathbf{1 2}$ years & Total \\
\hline Platelet classification & & & & \\
Normal & 37 & 15 & 4 & 53 \\
Thrombocytopenia & 17 & 12 & 2 & 33 \\
Thrombocytosis & 5 & 7 & & 14 \\
Malaria Type & & & 4 & 80 \\
Uncomplicated & 52 & 24 & 3 & 20 \\
Complicated & 7 & 10 & &
\end{tabular}

8 years was 10 and 9 to 12 years was 3 . Complicated malaria was seen to be more frequent among lesser age group below 9 years and this age groups 1 to 8 accounted for $85 \%$ of all the complicated cases.

\subsection{Prevalence of Malaria Infection and Platelet numbers According to Types and Based on Classification Respectively}

Table 2 shows the prevalence of malaria infection and platelet numbers according to types and based on classification respectively. Mild and moderate malaria infection was categorized as Uncomplicated and it accounted for 80\% (80) of the studied participants whiles Complicated accounted for $20 \%$ (20). Platelet numbers were also categorized into normal, thrombocytosis and thrombocytopenia based on the reference range. 53 children (53\%) had normal platelet numbers, 33 (33\%) had thrombocytopenia, and 14 children (14\%) had thrombocytosis.

\subsection{Prevalence of Malaria Types in Children with Thrombocytopenia}

Table 3 shows the prevalence of malaria types among children with thrombocytopenia. Out of the 33 (33\%) patients who had thrombocytopenia, $15(45.5 \%)$ of them had uncomplicated malaria and 18 (54.5\%) of them had complicated or severe malaria.

\subsection{Distribution and Prevalence of Platelet Numbers in Uncomplicated Malaria}

Table 4 shows classification of platelet numbers and their prevalence among children with uncomplicated (mild to moderate) malaria. Out the $80(80 \%)$ patients who had uncomplicated malaria, 15 (18.8\%) children had thrombocytopenia, $51(63.8 \%)$ had normal platelet numbers and 14 (17.5\%) children had thrombocytosis.

\subsection{Distribution and Prevalence of Platelet Numbers in Complicated/Severe Malaria}

Table 5 also shows the distribution and prevalence of platelet numbers among children with complicated or severe malaria. In all the 20 children diagnosed 
Table 2. Prevalence of malaria infection and platelet numbers according to types and based on classification respectively.

\begin{tabular}{ccccc}
\hline Category & Frequency & Percent (\%) & $\begin{array}{c}\text { Valid Percent } \\
(\%)\end{array}$ & $\begin{array}{c}\text { Cumulative } \\
\text { Percent (\%) }\end{array}$ \\
\hline Malaria Types & & & & \\
Uncomplicated & 8080 & 20 & 80 & 80 \\
Complicated & 20 & 100 & 100 & 100 \\
Total & 100 & & & \\
Plateles Classification & & 53 & 53 & 53 \\
$\quad$ Normal & 53 & 33 & 33 & 33 \\
Thrombocytopenia & 33 & 14 & 14 & 14 \\
Thrombocytosis & 14 & 100 & 100 & 100 \\
Total & 100 & & & \\
\hline
\end{tabular}

Table 3. Prevalence of malaria types in children with thrombocytopenia.

\begin{tabular}{ccccc}
\hline Category & Frequency & Percent (\%) & $\begin{array}{c}\text { Valid Percent } \\
(\%)\end{array}$ & $\begin{array}{c}\text { Cumulative } \\
\text { Percent (\%) }\end{array}$ \\
\hline Malaria Types & & & & \\
Uncomplicated & 15 & 45.5 & 45.5 & 45.5 \\
Complicated & 13 & 54.5 & 54.5 & 54.5 \\
Total & 33 & 100 & 100 & 100 \\
\hline
\end{tabular}

Table 4. Distribution and prevalence of platelet numbers in uncomplicated malaria.

\begin{tabular}{ccccc}
\hline Category & Frequency & $\begin{array}{c}\text { Percent } \\
(\%)\end{array}$ & $\begin{array}{c}\text { Valid Percent } \\
(\%)\end{array}$ & $\begin{array}{c}\text { Cumulative } \\
\text { Percent (\%) }\end{array}$ \\
\hline Plateles Classificatiion & & & & \\
Normal & 51 & 63.8 & 63.8 & 63.8 \\
Thrombocytopenia & 15 & 18.8 & 18.8 & 18.8 \\
Thrombocytosis & 14 & 17.5 & 17.5 & 17.5 \\
Total & 80 & 100 & 100 & 100 \\
\hline
\end{tabular}

Table 5. Distribution and prevalence of platelet numbers in complicated/severe malaria.

\begin{tabular}{ccccc}
\hline Category & Frequency & Percent (\%) & $\begin{array}{c}\text { Valid Percent } \\
(\%)\end{array}$ & $\begin{array}{c}\text { Cumulative } \\
\text { Percent (\%) }\end{array}$ \\
\hline Plateles Classificatiion & & & & \\
Normal & 2 & 10.0 & 10.0 & 10.0 \\
Thrombocytopenia & 18 & 90.0 & 90.0 & 90.0 \\
Thrombocytosis & 0 & 0.0 & 0.0 & 0.0 \\
Total & 20 & 100 & 100 & 100 \\
\hline
\end{tabular}

with complicated malaria, 18 (90\%) of them were found to have thrombocytopenia whiles $2(10 \%)$ of them were found to have normal platelet numbers. No child was found to have thrombocytosis during complicated malaria.

\section{Discussion}

Malaria is a Protozoal disease caused by infection with parasites of the genus 
Plasmodium and transmitted to man by certain species of infected female Anopheline mosquito, Haematological abnormalities have been observed in patients with malaria [20] but much attention has been placed on haemoglobin as the key indicator of relevance in relation to malaria infection in children with little or no regards to other haematological parameters including platelets. Malaria infection, especially an infection with Plasmodium falciparum is hazardous and deadly in children whose immune system is generally compromised, suppressed or incompetent. Thrombocytopenia is a common finding in malaria in children, and though it is a useful indicator of the disease, its prognostic value is not being addressed. In severe cases of the malaria, children presenting with a platelet count of less than $100 \times 10^{9} / \mathrm{L}$ are more likely to die because multivariate analysis identifies thrombocytopenia as an independent predictor of death [21]. Studies of malaria and thrombocytopenia began as early as 1967 where Dennis and Inman [22] studied depletion of coagulation factors in P. falciparum malaria and found out that platelet numbers tend to decrease somehow during the disease. In the 100 malaria patients studied, $61 \%$ were males and $39 \%$ were females. However, in assessing their platelet numbers, it was observed that the males had more normal platelet numbers than the females and at the same time were also the ones with more thrombocytopenia than the females. However, with thrombocytosis, the females had higher numbers than the male. This is in contrast to the literature reviewed which indicates that the parameters are unaffected by gender, the findings in this paper could be the results of the uneven nature of the male to female ratio in the sampling as there is no known correlation between gender and platelet count in malaria infections. Among the 33 malaria children with thrombocytopenia, 17 were between 1 to 4 years of age, 12 were between 5 to 8 years and only 4 were between 9 to 12 years. This also gives the impression that children under 5 years are more likely to develop thrombocytopenia during malaria as the effect of malaria is more severe in this group, [23] possibly owing to the fact that these children have not yet acquired the partial immunity to the disease that is usually acquired during childhood in high transmission areas [24]. Hence the positive association between lower ages and decreased platelet counts during malaria could be supported. Also, it could be deduced that children under 8 years have their malaria progressing to more severe forms than children above 9 years, again, as a result of undeveloped immunity in the absence of pathological suppression. 15 children with thrombocytopenia representing $45.5 \%$ had uncomplicated malaria whiles 18 children with thrombocytopenia, representing $54.5 \%$, had severe or complicated malaria. This can also be explained to indicate that there is a positive association between thrombocytopenia and severity of the malaria or the level of the parasitaemia of the infection. This finding is consistent with the data obtained by [21] which showed an association between thrombocytopenia and either severity or prognosis in childhood falciparum malaria infection. The prevalence of severe or complicated malaria was $20 \%$, whiles that of uncomplicated (mild to moderate) malaria was $80 \%$. This implies that most the 
children's condition had not yet progressed to its severity stage and is likely to have complicated malaria during the infection. It was also found that age groups 1 to 8 accounted for $85 \%$ of all the complicated cases, which further strengthens the deduction that children under 8 years are more likely to have their malaria progress to more severe forms. The overall prevalence of thrombocytopenia in children with malaria (not categorized) is $33 \%$ as compared to $53 \%$ for normal platelet numbers and $14 \%$ for thrombocytosis. This prevalence of thrombocytopenia closely agrees with the works done by [25] (22\%) and [6] (43\%). In mild to moderate forms of $P$. falciparum malaria, thrombocytopenia is not common because the disease has not yet progressed to its severe stage to cause depletion of the coagulation factors. This was observed in the study where thrombocytopenia in uncomplicated malaria gave a prevalence of $18.8 \%$ in 15 children. This is closely followed by thrombocytosis with $17.5 \%$ in 14 children. Half of the total number of children had normal platelet numbers, which represents $63.8 \%$. This implies that in non-severe forms of malaria, normal platelet numbers are more prevalent. In 1999, Kakar and his workers [8] carried out a research on malaria and thrombocytopenia and reported that $80 \%$ of the patients infected with either $P$. falciparum or $P$. vivax malaria develop thrombocytopenia during their infection, and it is more evident in those with the severe forms of the disease. Patel and his friends [26] also followed up on this in 2004 and concluded that thrombocytopenia complicates up to $60 \%$ to $80 \%$ cases of malaria, and its occurrence is a diagnostic clue for malaria in endemic areas. The main aim of this project was to determine the prevalence of thrombocytopenia among children with severe forms of malaria. 18 children with complicated or severe malaria had thrombocytopenia whiles only 2 of such children had normal platelet numbers. None of the children with severe malaria had thrombocytosis. These 18 children gave a prevalence of thrombocytopenia in severe malaria to be $90 \%$, and $10 \%$ for the normal platelet numbers. This thrombocytopenia prevalent result closely agrees with the works of [8] and [26] as stated previously. Moreover, [27] and [28] separately followed up on the reports of [8] and [26] in 2005 and 2010 respectively and came out respectively with a prevalence of thrombocytopenia in severe malaria children to be $85 \%$ and $90.9 \%$. These results strongly agree with that obtained in this project and it could therefore be deduced that thrombocytopenia is a useful indicator of malaria in children.

\section{Conclusion}

Platelet numbers decrease in malaria infection but very profound in severe malaria. The decrease is more evident in children below 5 years and slightly seen in children between 5 to 8 years. Platelet numbers are mostly normal in uncomplicated malaria and the detection of thrombocytosis in mild, moderate or severe malaria is insignificant. The prevalence of thrombocytopenia among children with severe malaria at the Korle Bu Teaching Hospital (KBTH) was found to be $90 \%$. It can therefore be concluded that mild to severe thrombocytopenia should 
alert the possibility of malaria infection and it is usually more common in Plasmodium falciparum malaria, the main species of malaria parasite in Ghana. The results also show that thrombocytopenia is more frequent and more profound in severe forms than mild to moderate forms. Whatever the clinical presentation, it is predictive of fatal death, and also an undetected and contributing factor to the deaths caused by malaria in children. In conclusion, the findings indicate a significant prognostic value of falciparum malaria-related thrombocytopenia among children in Ghana living in a high and seasonal transmission setting. The role of platelets in the pathogenesis of severe malaria should be further investigated in future studies.

\section{Limitations}

One of the limitations was our inability to get some guardians and parents' consent to the study. Another challenge was our limitation to rely solely on the subject's clinical and family history to rule out some disease conditions in order to recruit children suffering from malaria only. Notwithstanding that, the findings depict true reflection of the target population of children suffering from malaria alone.

\section{Acknowledgements}

We acknowledge the School of Allied Health Sciences of the University of Ghana, Department of Child Health and the Polyclinic of the Korle-Bu Teaching Hospital for allowing us to use their facilities to conduct this study.

\section{Conflicts of Interest}

We say on authority that there was no competing interest, as the study did not get any funding from any organization.

\section{Author's Contribution}

DMA and MHA designed and conducted the study and contributed in the preparation and drafting of the manuscript. AFS, JBA and SA participated in data interpretation, contributed to the performing the statistical analysis and the manuscript drafting. All authors read and approved the final manuscript.

\section{Availability of Data and Materials}

The supporting data for this study is available on request but restrictions apply to the availability of the data. Data are however available from the authors upon reasonable request and with permission from the institutions and facilities that were involved.

\section{Ethical Approval and Consent to Participate}

Approval for this study was obtained from the Ethical and Protocol Review Committee of the School of Allied Health Sciences of the University of Ghana, 
and the heads of the Department of Child Health and the Polyclinic of the Korle-Bu Teaching Hospital.

\section{References}

[1] Andrej, T., Matjaz, J., Igor, M. and Rajesh, P. (2003) Clinical Review: Severe Malaria. Journal of Critical Care, 7, 314-323.

[2] Greenwood, B.M., Boding, K., Witty, C.J. and Target, G.A. (2005) Malaria. Lancet, 365, 1487-1498. https://doi.org/10.1016/S0140-6736(05)66420-3

[3] Maina, R.N., Walsh, D., Gaddy, C., et al. (2010) Impact of Plasmodium falciparum Infection on Haematological Parameters in Children Living in Western Kenya. Malaria Journal, 9, Article No. S4. https://doi.org/10.1186/1475-2875-9-S3-S4

[4] Ndugwa, R.P., Muller, O., Kouyate, B., Bector, H. and Ramroth, H. (2007) Improving Malaria Mortality Estimates for Rural Africa. International Journal of Epidemiology, 36, 242-243. https://doi.org/10.1093/ije/dyl259

[5] Snow, R.W., Sartorius, B., Kyalo, D., Maina, J., Amratia, P., Mundia, C.W., Bejon, P. and Noor, A.M. (2017) The Prevalence of Plasmodium falciparum in Sub-Saharan Africa since 1900. Nature, 550, 515-518. https://doi.org/10.1038/nature24059

[6] Moulin, F., Lesage, F., Legros, A.H., et al. (2003) Thrombocytopenia and Plasmodium falciparum Malaria in Children with Different Exposures. Archives of Disease in Childhood, 88, 540-541. https://doi.org/10.1136/adc.88.6.540

[7] Lovareesuwan, S., Davis, J.G., Allen, D.L., Lee, S.H., Bunnag, D. and White, N.J. (1992) Thrombocytopenia in Malaria. Southeast Asian Journal of Tropical Medicine and Public Health, 23, 44-50.

[8] Kakar, A., Bhoi, S., Prakas, V. and Kakar, S. (1999) Profound Thrombocytopenia in Plasmodium Malaria. Diagnostic Microbiology and Infectious Disease, 35, 243-244. https://doi.org/10.1016/S0732-8893(99)00069-3

[9] Faseela, T.S., et al. (2011) Diagnostic Value of Platelet Count in Malaria. Journal of Clinical and Diagnostic Research, 5, 464-466.

[10] World Health Organization (2000) Severe Falciparum Malaria. Transactions of The Royal Society of Tropical Medicine and Hygiene, 94, S1/1-S1/90. https://doi.org/10.1016/S0035-9203(00)90300-6

[11] Jadhav, U.M., Patkar, V.S. and Kadam, N.N. (2004) Thrombocytopenia in Malaria-Correlation with Type and Severity of Malaria. JAPI, 52, 615-618.

[12] Kochar, D.K., Das, A., Kochar, A., et al. (2010) Thrombocytopenia in Plasmodium falciparum, Plasmodium vivax and Mixed Infection Malaria: A Study from Bikaner (Northwestern India). Platelets, 21, 623-627. https://doi.org/10.3109/09537104.2010.505308

[13] Grau, G.E., MacKenzie, C.D., Carr, R.A., et al. (2003) Platelet Accumulation in the Brain Microvessels in Fatal Pediatric Cerebral Malaria. The Journal of Infectious Diseases, 187, 461-466. https://doi.org/10.1086/367960

[14] Pain,A., Ferguson, D., Kai, O., et al. (2001) Platelet-Mediated Clumping of Plasmodium falciparum Infected Erythrocytes. Proceedings of the National Academy of Sciences of the United States of America, 98, 1805-1810. https://doi.org/10.1073/pnas.98.4.1805

[15] Newton, P., Essien, E. and White, N.J. (2004) Platelet and Blood Coagulation in Human Malaria. In: Abdallah, S.H. and Pavol, G., Eds., Malaria: A Haematological Perspective, Imperial College Press, London, 249-274.

https://doi.org/10.1142/9781860945908 0008 
[16] Kelton, J.G., Keystone, J., Moore, J., et al. (1983) Immune-Mediated Thrombocytopenia of Malaria. Journal of Clinical Investigation, 71, 832-836. https://doi.org/10.1172/JCI110836

[17] Ansari, S., Khoharo, H.K., Abro, A., Akhund, I.A. and Qureshi, F. (2009) Thrombocytopenia in Plasmodium falciparum Malaria. Journal of Ayub Medical College Abbottabad, 21, 145-147.

[18] Ghana Malaria Indicator Survey (GMIS) (2016) The DHS Program. https://dhsprogram.com/pubs/pdf/MF20/MF20.pdf

[19] Sumbele, I.U.N., Sama, S.O., Kimbi, H.K. and Taiwe, G.S. (2016) Malaria, Moderate to Severe Anaemia, and Malarial Anaemia in Children at Presentation to Hospital in the Mount Cameroon Area: A Cross-Sectional Study. Anemia, 2016, Article ID: 5725634. https://doi.org/10.1155/2016/5725634

[20] Gupta, N.K., Bansal, S.B., Jain, U.C. and Sahare, K. (2013) Study of Thrombocytopenia in Patients of Malaria. Tropical Parasitology, 3, 58-61. https://doi.org/10.4103/2229-5070.113914

[21] Gérardin, P., Rogier, C., Ka, A.S., Jouvencel, P., Brousse, V. and Imbert, P. (2002) Prognostic Value of Thrombocytopenia in African Children with Falciparum Malaria. The American Journal of Tropical Medicine and Hygiene, 66, 686-691. https://doi.org/10.4269/ajtmh.2002.66.686

[22] Dennis, L.H., Eichelberger, J.W. and Inman, M.M. (1967) Depletion of Coagulation Factors in Drug Resistant Plasmodium falciparum Malaria. Blood, 29, 713-721.

[23] Adedapo, A.D., Falade, C.O., Kotila, T. and Ademowo, O.G. (2007) Age as a Risk Factor for Thrombocytopenia and Anaemia in Children. Journal of Vector Borne Diseases, 44, 266-271.

[24] WHO (2018) Malaria in Children under Five. https://www.who.int/malaria/areas/high risk groups/children/en/

[25] Utta, M.K., Devrajani, B.R., Shaik, K., Shaik, K.R. and Shah, S.Z. (2010) Severity of Thrombocytopenia and Prolonged Bleeding Time in Patients with Malaria. World Applied Sciences Journal, 9, 484-488.

[26] Patel, U., Gandhi, G., Friedman, S. and Niranjan, S. (2004) Thrombocytopenia in Malaria. Journal of the National Medical Association, 6, 1212-1214.

[27] Horstmann, R., Dietrich, M. and Rasche, H. (2005) Malaria-Induced Thrombocytopenia. Annals of Hematology, 42, 157-164.

[28] Leowattana, W., Tangpukdee, N., Thar, S.K., Nakasiri, S., Srivilairit, S., Kano, S., et al. (2010) Changes in Platelet Count in Uncomplicated and Severe Falciparum Malaria. Southeast Asian Journal of Tropical Medicine and Public Health, 41, 1035. 